\title{
THE COHOMOLOGY OF A SUBALGEBRA OF THE STEENROD ALGEBRA
}

\author{
BY \\ ARUNAS L. LIULEVICIUS( $\left.{ }^{1}\right)$
}

1. Introduction. Let $A$ be the Steenrod algebra over $Z_{p}, p$ a prime. It is well known [4] that it is an augmented Hopf algebra over $Z_{p}$. The groups $\operatorname{Ext}_{A}^{s, t}\left(Z_{p}, Z_{p}\right)$ occur as the $E_{2}$ term of a spectral sequence which may be used to determine the $p$-primary components of stable homotopy groups of spheres [1]. Recent results of Adams [3] indicate that it is fruitful to study the groups $\operatorname{Ext}_{B}\left(Z_{p}, Z_{p}\right)$, where $B$ is a Hopf subalgebra of $A$. In this paper we study a certain subalgebra of the Steenrod algebra. The results obtained here will be used in proving an Adams periodicity theorem for $p$ an odd prime. The fundamental tool in this investigation is the twisted tensor product construction introduced by Wall [5].

2. A Hopf algebra on two generators. Let $W$ be a graded, connected algebra with unit and augmentation, generated as an algebra over $Z_{p}$ ( $p$ a prime) by two elements: $x$ of grade $q, z$ of grade $p q$, satisfying the following relations:

$$
\begin{aligned}
x[z, x] & =[z, x] x, \\
z[z, x] & =[z, x] z, \\
x^{p} & =0, \\
z^{p} & =([z, x])^{p-1} x,
\end{aligned}
$$

where $[z, x]=z x-x z$.

For $p=2$ we may interpret $W$ as the subalgebra of the Steenrod algebra generated by $S q^{1}$ and $S q^{2}$, by letting $q=1, x=S q^{1}, z=S q^{2}$. For $p$ odd, we let $x=P^{1}, z=P^{p}, q=2 p-2:$ in this case $W$ is again the subalgebra of the Steenrod algebra generated by $P^{1}$ and $P^{p}[4]$.

Let us write $y$ for $[z, x]$. It is an easy consequence of the above relations that $y^{p}=0$.

Let $V$ be the subalgebra of $W$ generated by $1, x$ and $y$. Since $x$ and $y$ commute, and $x^{p}=y^{p}=0, V$ is, as a (Hopf) algebra, just a tensor product of two truncated polynomial algebras. Thus a minimal resolution for $Z_{p}$ over $V$ can be taken to be a tensor product of two minimal resolutions of $Z_{p}$ over truncated polynomial algebras. We thus have

$$
\begin{aligned}
\operatorname{Ext}_{V}^{*, *}\left(Z_{p}, Z_{p}\right) & \cong \mathscr{L}\left\{h_{0}, \lambda_{0}, h_{2,0}, \lambda_{2,0}\right\} & & \text { if } p \text { odd } \\
& \cong Z_{2}\left[h_{0}, h_{2,0}\right] & & \text { if } p=2
\end{aligned}
$$

Received by the editors October 24, 1961.

(1) The author holds a National Science Foundation Postdoctoral Fellowship. 
where $\mathscr{L}\{\}$ denotes the free associative, commutative bigraded algebra over $Z_{p}$ on generators

$$
\begin{array}{ll}
h_{0} \text { of bidegree } & (1, q), \\
h_{2,0} \text { of bidegree } & (1, q(p+1)), \\
\lambda_{0} \text { of bidegree } & (2, p q), \\
\lambda_{2,0} \text { of bidegree } & (2, p q(p+1)) .
\end{array}
$$

A minimal resolution for $Z_{p}$ over $V$ is given by the following complex $\mathcal{F}$ :

$$
0 \leftarrow Z_{p} \stackrel{\epsilon_{F}}{\leftarrow} F_{0} \leftarrow F_{1} \leftarrow \cdots \leftarrow F_{s} \stackrel{d_{F}}{\leftarrow} F_{s+1} \leftarrow \cdots .
$$

We take $F_{s}=V \otimes_{Z_{p}} \bar{F}_{s}$, where $\bar{F}_{s} \cong \operatorname{Ext}_{V}^{s, *}\left(Z_{p}, Z_{p}\right)$. We thus conveniently confuse the generators of $F_{s}$ with elements of a $Z_{p}$ basis of $\operatorname{Ext}_{V}^{s, *}\left(Z_{p}, Z_{p}\right)$.

We take as a basis for $\bar{F}_{8}$ the set of formal monomials $h_{0} \lambda_{0}^{n} h_{2,0}^{\eta} \lambda_{2,0}^{r}$, where $\epsilon, \eta=1$ or 0 , and $\epsilon+2 n+\eta+2 r=s$. If $p=2$ we omit $\lambda_{2,0}$ and substitute $h_{2,0}^{2}$ for every occurrence of $\lambda_{2,0}$. We define the differential $d_{F}$ in $\mathcal{F}$ as follows:

$$
\begin{aligned}
d_{F}\left(\lambda_{0}^{n} \lambda_{2,0}^{r}\right) & =x^{p-1} h_{0} \lambda_{0}^{n-1} \lambda_{2,0}^{r}+y^{p-1} \lambda_{0}^{n} h_{2,0} \lambda_{2,0}^{r-1}, \\
d_{F}\left(h_{0} \lambda_{0}^{n} \lambda_{2,0}^{r}\right) & =x \lambda_{0}^{n} \lambda_{2,0}^{r}-y^{p-1} h_{0} \lambda_{0}^{n} h_{2,0} \lambda_{2,0}^{r-1}, \\
d_{F}\left(\lambda_{0}^{n} h_{2,0} \lambda_{2,0}^{r}\right) & =x^{p-1} h_{0} \lambda_{0}^{n-1} h_{2,0} \lambda_{2,0}^{r}+y \lambda_{0}^{n} \lambda_{2,0}^{r}, \\
d_{F}\left(h_{0} \lambda_{0}^{n} h_{2,0} \lambda_{2,0}^{r}\right) & =x \lambda_{0}^{n} h_{2,0} \lambda_{2,0}^{r}-y h_{0} \lambda_{0}^{n} \lambda_{2,0}^{r},
\end{aligned}
$$

where we set $\alpha^{k}=1$ if $k=0, \alpha^{k}=0$ if $k<0$.

We notice that $V$ is a normal subalgebra of $W: \bar{V} W=W \bar{V}$, where $\bar{V}$ is the augmentation ideal. This follows from the relations

$$
\begin{aligned}
& z x=x z+y, \\
& z y=y z .
\end{aligned}
$$

The normal quotient $U=W / / V=W / W \bar{V}$ is clearly a truncated polynomial algebra on the residue class of $z$, denoted $\bar{z}$. We know that

$$
\operatorname{Ext}_{U}^{*, *}\left(Z_{p}, Z_{p}\right) \cong \mathscr{L}\left\{h_{1}, \lambda_{1}\right\}
$$

where $h_{1}$ is of bidegree $(1, p q)$ and $\lambda_{1}$ is of bidegree $\left(2, p^{2} q\right)$, and where (once more) if $p=2$ we omit $\lambda_{1}$ and substitute $h_{1}^{2}$ for every occurrence of $\lambda_{1}$. We let Q be the following minimal resolution of $Z_{p}$ over $U$ :

$$
0 \leftarrow Z_{p} \stackrel{\epsilon_{B}}{\leftarrow} B_{0} \leftarrow B_{1} \leftarrow \cdots \leftarrow B_{r} \stackrel{d_{B}}{\leftarrow} B_{r+1} \cdots,
$$

where $B_{r}=U \otimes_{z_{p}} \bar{B}_{r}$, and $\bar{B}_{r}$ is 1 -dimensional over $Z_{p}$, with a basis $h_{1} \lambda_{1}^{k}$, where $\epsilon=0$ or 1 , and $\epsilon+2 k=r$. 
We let

$$
\begin{aligned}
& d_{B}\left(h_{1} \lambda_{1}^{k}\right)=\bar{z} \lambda_{1}^{k}, \\
& d_{B}\left(\lambda_{1}^{k+1}\right)=\bar{z}^{p-1} h_{1} \lambda_{1}^{k} .
\end{aligned}
$$

Let $\varepsilon$ be the following (three-graded) free $W$-module:

$$
\begin{aligned}
\mathcal{E}_{r, s} & =W \otimes_{z_{p}} \bar{B}_{r} \otimes_{z_{p}} \bar{F}_{s}, \\
\mathcal{E} & =\sum_{r, s} \mathcal{E}_{r, s .} .
\end{aligned}
$$

We introduce an augmentation

$$
\epsilon_{8}: \mathcal{E}_{s, *} \rightarrow B_{s}
$$

by setting $\epsilon_{s}=\pi \otimes \epsilon_{F}$, where $\pi$ is the projection

$$
\pi: W \rightarrow U, \text { and } \epsilon_{F} \text { is the augmentation in } \mathcal{F} .
$$

3. Twisted tensor product of resolutions. Consider the following situation (we have described a special case of it above). Let $W, U, V$ be augmented algebras over a field $K$. Let $B, \mathcal{F}$ be free resolutions of $K$ over $U, V$, respectively. Let $\bar{B}, \bar{F}$ be the $K$-complexes" $K \otimes_{U} B, K \otimes_{V_{-}}$'F.

TheOREM 1 (WALL). If (1) $V$ is normal in $W$, (2) $W$ is free as a right module over $V$, (3) $U=W / / V=W / W \bar{V}$, then a free resolution $\&$ of $K$ over $W$ can be constructed as a twisted tensor product of $\overline{\mathbb{B}}$ and $\bar{F}$. That is, if

$$
\begin{aligned}
\mathcal{E} & =\sum_{r, s} \mathcal{E}_{r, s}, \\
\mathcal{E}_{r, s} & =W \otimes_{K} \bar{B}_{r} \otimes_{K} \bar{F}_{s},
\end{aligned}
$$

then there exists an augmentation

$$
\epsilon: \mathcal{E} \rightarrow \mathbb{B}
$$

and $W$-maps

$$
d_{k}: \mathcal{E}_{r, 8} \rightarrow \mathcal{E}_{r-k, 8+k-1}
$$

such that

$$
\begin{aligned}
d_{0} & =1 \otimes 1 \otimes d_{F} \\
\epsilon d_{1} & =d_{B} \epsilon
\end{aligned}
$$

and

$$
\sum_{j=0}^{k} d_{k-j} d_{j}=0 \quad \text { for } k=0,1, \cdots
$$


Proof. We take the proof of Wall [5] for the special case $W=Z(G)$, $U=Z(H), V=Z(K)$, where $G, H, K$ are finite groups, $K \triangleleft G, H=G / K$. If we substitute $W, U, V$ for $Z(G), Z(H), Z(K)$, respectively, wherever they appear in Wall's proof, the result is a proof of Theorem 1.

We make $\varepsilon$ in to a complex by setting $d_{E}=\sum_{j=0}^{\infty} d_{j}$.

We remark that the hypothesis (2) of the theorem is always satisfied if $V$ is a Hopf subalgebra of the Hopf algebra $W$.

Proposition 1. If $W, V, U, \mathcal{E}$ are as in $\$ 2$, we let $\gamma_{q}$ be the chosen basis element of $\bar{B}_{q}$. We can define the maps $d_{k}$ as follows:

(1) $d_{0}$ is induced by $d_{F}$,

(2) if $q$ is an even integer,

$$
\begin{aligned}
d_{1}\left(\gamma_{q+1} \otimes \lambda_{0}^{n} \lambda_{2,0}^{r}\right) & =z \gamma_{q} \otimes \lambda_{0}^{n} \lambda_{2,0}^{r}-x^{p-2} \gamma_{q} \otimes h_{0} \lambda_{0}^{n-1} h_{2,0} \lambda_{2,0}^{r}, \\
d_{1}\left(\gamma_{q+1} \otimes \lambda_{0}^{n} h_{2,0} \lambda_{2,0}^{r}\right) & =z \gamma_{q} \otimes \lambda_{0}^{n} h_{2,0} \lambda_{2,0}^{r}, \\
d_{1}\left(\gamma_{q+1} \otimes h_{0} \lambda_{0}^{n} \lambda_{2,0}^{r}\right) & =z \gamma_{q} \otimes h_{0} \lambda_{0}^{n} \lambda_{2,0}^{r}-\gamma_{q} \otimes \lambda_{0}^{n} h_{2,0} \lambda_{2,0}^{r}, \\
d_{1}\left(\gamma_{q+1} \otimes h_{0} \lambda_{0}^{n} h_{2,0} \lambda_{2,0}^{r}\right) & =z \gamma_{q} \otimes h_{0} \lambda_{0}^{n} h_{2,0} \lambda_{2,0}^{r},
\end{aligned}
$$

(3) $d_{2}\left(\gamma_{q+2} \otimes \alpha\right)=(-1)^{q+1} y^{p-2} x \gamma_{q} \otimes h_{2,0} \alpha$, where $q$ is a non-negative integer, and $\alpha$ is a basic monomial,

(4) $d_{2 m}=0$ identically for $m \geqq 2$,

(5) $d_{3}\left(\gamma_{q+3} \otimes \alpha\right)=(r+1) \gamma_{q} \otimes \alpha \lambda_{2,0}$, where $q$ is an even integer and $\alpha$ is a basic monomial of the form $h_{0}^{\epsilon} \lambda_{0}^{n} h_{2,0}^{\dot{\eta}} \lambda_{2,0}^{r}$,

(6) $d_{2 m+1}\left(\gamma_{q+2 m+1} \otimes \alpha\right)$ for $q$ odd is defined as follows:

$$
\begin{aligned}
& d_{2 m+1}\left(\lambda_{1}^{k+m+1} \otimes \lambda_{0}^{n} \lambda_{2,0}^{r}\right)=(-1)^{m} m !\left(\begin{array}{c}
r+m \\
r
\end{array}\right) z^{p-m-1} h_{1} \lambda_{1}^{k} \otimes \lambda_{0}^{n} \lambda_{2,0}^{r+m} \\
&+(-1)^{m+1}\left(\begin{array}{c}
r+m \\
r
\end{array}\right) \sum_{j=1}^{p-m-1}(-1)^{j}(j+m) ! z^{p-m-1-j} y^{j-1} x^{p-1-j} h_{1} \lambda_{1}^{k} \otimes h_{0} \lambda_{0}^{n-1} h_{2,0} \lambda_{2,0}^{r+m}, \\
& d_{2 m+1}\left(\lambda_{1}^{k+m+1} \otimes \lambda_{0}^{n} h_{2,0} \lambda_{2,0}^{r}\right)=(-1)^{m} m !\left(\begin{array}{c}
r+m \\
r
\end{array}\right) z^{p-m-1} h_{1} \lambda_{1}^{k} \otimes \lambda_{0}^{n} h_{2,0} \lambda_{2,0}^{r+m}, \\
& d_{2 m+1}\left(\lambda_{1}^{k+m+1} \otimes h_{0} \lambda_{0}^{n} \lambda_{2,0}^{r}\right)=(-1)^{m} m !\left(\begin{array}{c}
r+m \\
r
\end{array}\right) z^{p-m-1} h_{1} \lambda_{1}^{k} \otimes h_{0} \lambda_{0}^{n} \lambda_{2,0}^{r+m} \\
&+(-1)^{m}(m+1) !\left(\begin{array}{c}
r+m \\
r
\end{array}\right) z^{p-m-2} h_{1} \lambda_{1}^{k} \otimes \lambda_{0}^{n} h_{2,0} \lambda_{2,0}^{r+m},
\end{aligned}
$$$$
d_{2 m+1}\left(\lambda_{1}^{k+m+1} \otimes h_{0} \lambda_{0}^{n} h_{2,0} \lambda_{2,0}^{r}\right)=(-1)^{m} m !\left(\begin{array}{c}
r+m \\
r
\end{array}\right) z^{p-m-1} h_{1} \lambda_{1}^{k} \otimes h_{0} \lambda_{0}^{n} h_{2,0} \lambda_{2,0}^{r+m}
$$

(7) $d_{2 m+1}\left(\gamma_{q+2 m+1} \otimes \alpha\right)=0$ if $q$ is even and $m \geqq 2$. 
Proof. Easy, but tedious induction on the total degree. Special care must be given for $d_{j}, j \leqq 6$.

4. Structure of $\operatorname{Ext}_{W}\left(Z_{p}, Z_{p}\right)$. In order to determine the additive structure of $\operatorname{Ext}_{W}\left(Z_{p}, Z_{p}\right)$, it is sufficient to compute $\operatorname{Tor}^{W}\left(Z_{p}, Z_{p}\right)$, since the first is the graded dual of the second [2]. We exhibit certain elements of $\operatorname{Ext}_{W}\left(Z_{p}, Z_{p}\right)$ in the following table (here $p \neq 2$; the structure of $\operatorname{Ext}_{W}\left(Z_{2}, Z_{2}\right)$ is given in Theorem 3). The first column gives the element in Ext which is the dual of the corresponding element in the third column (this makes sense, for in each relevant grading $\operatorname{Tor}^{W}\left(Z_{p}, Z_{p}\right)$ turns out to be 1-dimensional).

TABLE $1(p \neq 2)$

\begin{tabular}{llc}
\hline \hline Class in Ext & Bidegree & Representative in Tor \\
\hline$h_{0}$ & $(1, q)$ & $1 \otimes h_{0}$ \\
$h_{1}$ & $(1, p q)$ & $h_{1} \otimes 1$ \\
$\lambda_{0}$ & $(2, p q)$ & $1 \otimes \lambda_{0}$ \\
$\lambda_{1}$ & $\left(2, p^{2} q\right)$ & $\lambda_{1} \otimes 1$ \\
$\mu_{0}$ & $(2,(p+2) q)$ & $\frac{1}{2} 1 \otimes h_{0} h_{2,0}$ \\
$\nu_{0}$ & $(2,(2 p+1) q)$ & $\frac{1}{3} h_{1} \otimes h_{2,0}$ \\
$\chi$ & $\left(3,\left(p^{2}+p+1\right) q\right)$ & $1 \otimes h_{0} \lambda_{2,0}$ \\
$\sigma_{2 r+1} 1 \leqq r<p-1$ & $\left(2 r+1,\left(r p^{2}+r p+p\right) q\right)$ & $h_{1} \otimes \lambda_{2,0}^{r}$ \\
$\kappa_{2 s} 2 \leqq s<p-1$ & $\left(2 s,\left(s p^{2}-p^{2}+s p+p+1\right) q\right)$ & $h_{1} \otimes h_{2,0}^{r-1}$ \\
$\omega$ & $\left(2 p, p^{2}(p+1) q\right)$ & $1 \otimes \lambda_{2,0}^{p}$ \\
\hline
\end{tabular}

THEOREM $2(p \neq 2)$. The classes $\lambda_{0}$ and $\omega$ generate a free associative, commutative algebra $L$ in $\operatorname{Ext}_{W}\left(Z_{p}, Z_{p}\right)$. The classes $\lambda_{1}^{k} 0 \leqq k<p, h_{0} \lambda_{1}^{q} 0 \leqq q<p-1$, $\mu_{0} \lambda_{1}^{k} 0 \leqq k<p, \chi \lambda_{1}^{k} 0 \leqq k<p, \nu_{0}, h_{0} \nu_{0}, \sigma_{2 r+1} 1 \leqq r<p-1, h_{0} \sigma_{2 r+1} 1 \leqq r<p-1$, $\kappa_{2 s} 2 \leqq s<p-1, h_{0} \kappa_{2 s} 2 \leqq s<p-1, \mu_{0} \sigma_{2 r+1} 1 \leqq r<p-1$ is a system of generators for $\operatorname{Ext}_{W}\left(Z_{p}, Z_{p}\right)$ as a free left L-module.

Proof. We first remark that the elements $1 \otimes \lambda_{0}$ and $1 \otimes \lambda_{2,0}^{p}$ indeed give rise to nonzero elements in $\operatorname{Ext}_{W}\left(Z_{p}, Z_{p}\right)$. Secondly, we notice that the resolution $\mathcal{E}$ of $Z_{p}$ is honestly periodic with respect to the formal multiplication of the generators by $\lambda_{0}$ and $\lambda_{2,0}^{p}$. It is now clear that the formal multiplication by $\lambda_{0}$ or $\lambda_{2,0}^{p}$ corresponds to honest multiplication by $\lambda_{0}$ or $\omega$ in $\operatorname{Ext}_{W}\left(Z_{p}, Z_{p}\right)$ : this is an immediate consequence of the preceding sentence and the Yoneda construction for the product (see, for example, p. 30 [2]). We now only have to verify that the listed classes indeed give an $L$-basis for $\operatorname{Ext}_{W}\left(Z_{p}, Z_{p}\right)$. This is indeed the case, for a basis for

$$
\operatorname{Ker} \bar{d} / \operatorname{Im} \bar{d}=\operatorname{Tor}^{W}\left(Z_{p}, Z_{p}\right)
$$

( $\bar{d}$ denotes $1 \otimes d$ in $Z_{p} \otimes_{W} \mathcal{E}$ ) is given by the cosets of the following elements: 


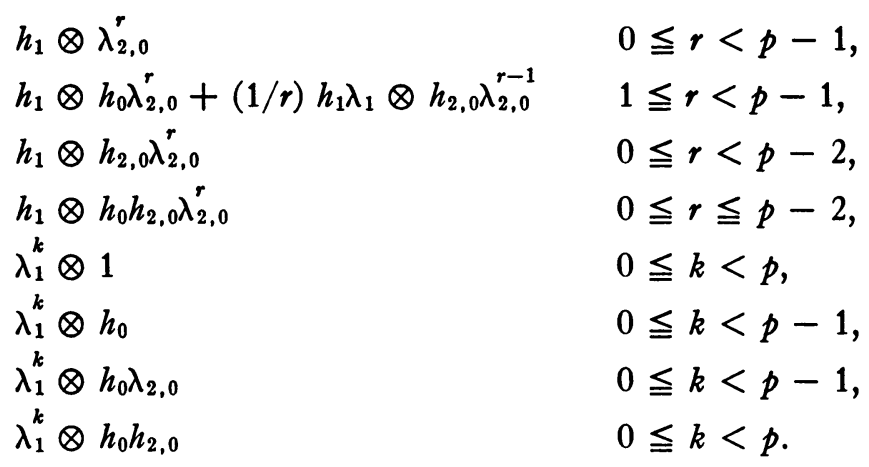

For completeness, we give the result for $p=2$ also. This is actually very easy: a direct proof via a minimal resolution is here painless.

Theorem $3(p=2)$. The algebra $\operatorname{Ext}_{W}\left(Z_{2}, Z_{2}\right)$ is isomorphic to the quotient of a polynomial algebra on classes

$$
\begin{aligned}
& h_{0} \text { of bigrading }(1, q) \\
& h_{1} \text { of bigrading }(1,2 q), \\
& u \text { of bigrading }(3,7 q), \\
& \omega \text { of bigrading }(4,12 q),
\end{aligned}
$$

modulo the ideal generated by the classes $h_{0} h_{1}, h_{1}^{3}, h_{1} u, u^{2}+h_{0}^{2} \omega$.

Proof. The reader is invited to construct a minimal resolution.

To give an idea of the algebra structure of $\operatorname{Ext}_{W}\left(Z_{p}, Z_{p}\right), p$ odd, we give the result for $p=3$ (in a way this is unfair, because here we have many more relations than for the general $p \geqq 5$ ).

ThEOREM $4(p=3)$. The indecomposable elements in $\operatorname{Ext}_{W}\left(Z_{3}, Z_{3}\right)$ have a basis consisting of the elements $h_{0}, \lambda_{0}, h_{1}, \lambda_{1}, \mu_{0}, \nu_{0}, \chi, \omega, \sigma_{3}$. The relations satisfied by these elements are (where $\{,$,$\} is the Massey triple product):$

$$
\begin{aligned}
h_{1} h_{0} & =0, & h_{1} \chi & =-h_{0} \sigma_{3}=\lambda_{1} \nu_{0}, \\
\lambda_{1} h_{1} & =0, & \lambda_{1} \sigma_{3} & =0, \\
\lambda_{1}^{2} h_{0} & =0, & h_{1} \lambda_{0} & =-h_{0} \mu_{0}, \\
\nu_{0} & =\left\{h_{1}, h_{0}, h_{1}\right\}, & \lambda_{1} h_{0} & =-h_{1} \nu_{0}, \\
\mu_{0} & =\left\{h_{0}, h_{1}, h_{0}\right\}, & h_{1} \sigma_{3} & =-\lambda_{1}^{2}, \\
\sigma_{3} & =\left\{h_{1}, \lambda_{1}, h_{1}\right\}, & h_{0} \chi & =\lambda_{1} \mu_{0}, \\
\chi & =\left\{h_{0}, h_{1}, \lambda_{1}\right\}, & \lambda_{0} \nu_{0} & =-\mu_{0}^{2}, \\
h_{1} \mu_{0} & =h_{0} \nu_{0}, & \lambda_{1} \chi & =\sigma_{3} \nu_{0} .
\end{aligned}
$$

Proof. We can exhibit a minimal resolution for $Z_{3}$ over $W$ and read off the above relations by means of the Yoneda constructions. 


\section{REFERENCES}

1. J. F. Adams, On the structure and applications of the Steenrod algebra, Comment. Math. Helv. 32 (1958), 180-214.

2. - On the non-existence of elements of Hopf invariant one, Ann. of Math. 72 (1960), 20-103.

3. - Stable homotopy theory (Mimeo. notes), University of California, Berkeley, Calif., 1961.

4. J. Milnor, The Steenrod algebra and its dual, Ann. of Math. 67 (1958), 150-171.

5. C. T. C. Wall, Resolutions for extensions of groups, Proc. Cambridge Philos. Soc. 57 (1961), 251-255.

The Institute for Advanced Study, Princeton, New Jersey

The University of Chicago, Chicago, Illinois 